\title{
Delayed sequence intubation: is it ready for prime time?
}

\author{
Reviewed by: John A. Taylor, MD, MPH*; Corinne Michele Hohl, MD, MHSc*
}

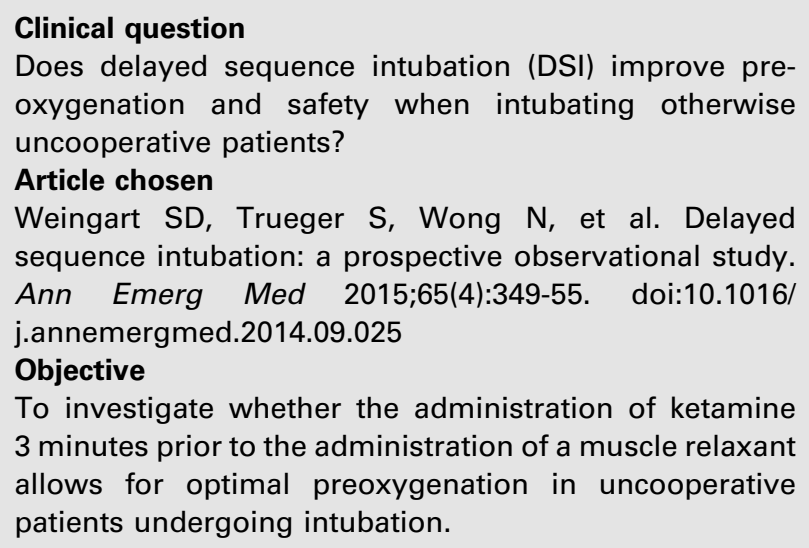

Keywords: intubation, sedation, ketamine, delayed sequence intubation

\section{BACKGROUND}

In patients who require urgent endotracheal intubation, optimal preoxygenation to create an oxygen reservoir in the lungs helps avoid desaturation during rapid sequence induction (RSI) ${ }^{1-4}$ However, patients who are uncooperative due to delirium, intoxication, or head trauma can be difficult to preoxygenate, because they might be noncompliant with the application of a face mask, attempts at delivering noninvasive positive pressure ventilation, or with other procedures.

Ketamine is a dissociative anesthetic that preserves respiratory drive and airway reflexes, while maintaining cardiovascular stability. ${ }^{5}$ In standard RSI, an induction agent such as ketamine is administered as an intravenous bolus, followed immediately by a muscle relaxant.
A period of apnea ensues while the muscle relaxant is taking effect, but during which optimal intubating conditions have not yet been achieved. Adequate preoxygenation ensures that patients do not desaturate without bag-mask-valve ventilation during this period, because this would increase the risk of gastric insufflation, vomiting, and aspiration.

Weingart et al. describe a procedure they coin delayed sequence intubation (DSI) in which an induction dose of ketamine is administered to sedate otherwise uncooperative patients for 3 minutes prior to the use of a muscle relaxant. ${ }^{6}$ They hypothesize that the early administration of ketamine may facilitate oxygen administration and procedures deemed necessary prior to RSI.

\section{POPULATION STUDIED}

The investigators enrolled a convenience sample of patients who required airway management, were at least 18 years old, breathing spontaneously, had no predictors of a difficult airway, and were uncooperative with at least three attempts of standard preoxygenation.

\section{STUDY DESIGN}

If three attempts at standard preoxygenation failed, investigators administered ketamine at a starting dose of $1 \mathrm{mg} / \mathrm{kg}$ followed by aliquots of $0.5 \mathrm{mg} / \mathrm{kg}$. They administered a muscle relaxant 3 minutes later. Patients served as their own controls, with investigators comparing oxygen saturations before administration and 3 minutes after administration of ketamine.

From the *Emergency Department, Vancouver General Hospital, Vancouver, BC.

Correspondence to: Dr. John A. Taylor, University of British Columbia, Department of Emergency Medicine, JPPN, Vancouver General Hospital, Room 3300, 910 West 10th Avenue, Vancouver, BC V5Z 1M9; Email: john.au.taylor@gmail.com, jataylo@ucalgary.ca 


\section{OUTCOME MEASURES}

The primary outcome was the difference between oxygen saturations at the time when the decision to proceed with DSI was made, compared with 3 minutes after ketamine was given, and prior to intubation. Secondary outcomes were the number of nasogastric tubes placed and the number of patients who tolerated high-flow oxygen for 3 minutes. The authors recorded complications, including apnea, emesis, and cardiac arrest.

Results were presented as descriptive statistics, and means were compared using paired T-test. A planned subgroup analysis included the difference in oxygen saturations pre- versus post-DSI in patients who were initially hypoxic.

\section{RESULTS}

Sixty-four patients were enrolled in the study, of which two were excluded after administration of ketamine because the investigators could not measure their oxygen saturations. Thirty-two patients had baseline oxygen saturations of less than $93 \%$. Patients required an average of $1.4 \mathrm{mg} / \mathrm{kg}$ of ketamine for dissociation.

Oxygen saturations increased from a mean of $89.9 \%$ pre-DSI to $99.8 \%$ post-DSI among all patients. Among the 32 hypoxic patients, all increased their oxygen saturations, and 29 had post-intervention saturations of greater than $93 \%$. Means and confidence intervals were not presented for this subgroup, but the raw data are presented in figure form. Four nasogastric tubes were placed, and no complications were reported.

\section{COMMENTARY}

This research describes the use of DSI by authors experienced in its use and is described as a prospective observational study. ${ }^{7}$ The authors report clinically and statistically significant increases in oxygen saturations after the administration of ketamine in otherwise uncooperative patients prior to intubation.

Investigations using single subjects as their own historical controls are called single-subject research designs and are a form of quasi-experimental investigation that may be used to evaluate treatment effectiveness in a single subject or a small group of subjects. ${ }^{8,9}$ In contrast, in experimental group designs, one group of individuals is exposed to an experimental intervention and compared with individuals who are not exposed. Ideally, subjects are randomly allocated to treatment assignment, and along with investigators blinded to treatment allocation.

When experimental methods such as randomized controlled trials are not feasible, or preliminary data are required to design more definitive studies, singlesubject designs may be considered. ${ }^{8,9}$ The key to establishing causality and optimizing internal validity using these designs is the use of multiple measurements in at least two phases of investigation (baseline and intervention) to ensure that the measurements are representative. ${ }^{8}$ Alternatively, repeat measurements in at least three phases of investigation may be completed, including measurements after completion of the intervention during which period the study subjects must remain at risk for the outcome being measured. As a rule of thumb, measuring a minimum of five data points minimizes the probability that the observed results are due to chance alone and the chance of selection bias that could occur if investigators chose the most advantageous time-point for measuring the study outcome, as well as the sequence of intervention. ${ }^{8}$

The reviewed study is intriguing. Yet, without the use of recommended methods to safeguard its internal validity, we recommend interpreting its results with caution. We believe that the use of an external control group that would have received a placebo infusion with continued attempts at preoxygenation (e.g., using application of nasal prongs and/or a dedicated provider using a two-handed technique to apply a face mask) may have helped isolate the effect of ketamine from the natural history of preoxygenating these patients. Alternatively, an external control group could undergo RSI with bag mask ventilation.

Another potential limitation is the use of a convenience sample of patients. In a trial, a clear description of all patients refusing participation is required, despite meeting inclusion and exclusion criteria. This helps assess whether and how selection bias may have influenced the reported outcomes and assists readers with assessing the study's external validity. Furthermore, although it is reassuring that the authors reported no complications, only 62 patients were included, and rare but serious complications may not have occurred in this small cohort. Enrolling this sample took 2.5 years at three major centres, indicating that this procedure would apply to a small group of patients or that selection bias was possible. Given these limitations, we 
believe it would be prudent to describe the study as a case series.

For decades, ketamine fell out of favor in North America because case-series indicated an association with increased intracranial pressure, a phenomenon now being questioned in systematic reviews. ${ }^{10,11}$ Although Weingart et al.'s case series shows promising results with no complications, it does not directly compare DSI to RSI, or to other methods of preoxygenating uncooperative patients. Given the known fallibility of case series and its small sample size, we believe that additional experimental research in this area is required prior to concurring with the authors' that "delayed sequence intubation seems safe and effective for use in emergency airway management." Although the surrogate outcome of pre-intubation oxygen saturation is relevant and the authors report other important outcomes, additional safety outcomes, including peri-intubation desaturation, hemodynamic instability, arrhythmias, and aspiration, need to be demonstrated in larger samples and compared to the current standard of care, before adopting DSI into routine practice.

\section{CONCLUSION}

In patients who are uncooperative and do not tolerate preoxygenation or required procedures, DSI may be safe and effective when applied by experienced practitioners. This case series describes an important innovation in airway management for a challenging patient population, but larger externally controlled studies are required to establish its effectiveness and safety prior to its adoption into routine practice.
Competing interests: None declared.

\section{REFERENCES}

1. Mort TC. Preoxygenation in critically ill patients requiring emergency tracheal intubation. Crit Care Med 2005; 33(11):2672-5 .

2. Tanoubi I, Drolet P, Donati F. Optimizing preoxygenation in adults. Can $\mathcal{F}$ Anesth 2009;56(6):449-66.

3. Walls RM, Murphy MF, eds. Manual of emergency airway management. 4th ed. Philadelphia: Lippincott Williams \& Wilkins; 2012.

4. Kovacs G, Law JA, eds. Airway management in emergencies, 2nd ed. Shelton. CT: People's Medical Publishing HouseUSA; 2011.

5. Green SM, Roback MG, Kennedy RM, et al. Clinical practice guideline for emergency department ketamine dissociative sedation: 2011 update. Ann Emerg Med 2011; 57(5):449-61.

6. Weingart SD, Trueger S, Wong N, et al. Delayed sequence intubation: a prospective observational study. Ann Emerg Med 2015;65(4):349-55.

7. Weingart SD. Preoxygenation, reoxygenation, and delayed sequence intubation in the emergency department. 7 Emerg Med 2011;40(6):661-7.

8. Backman CL, Harris SR. Case studies, single-subject research, and $\mathrm{N}$ of 1 randomized trials: comparisons and contrasts. Am 7 Phys Med Rebabil 1999;78(2):170-6.

9. Smith JD. Single-case experimental designs: a systematic review of published research and current standards. Psychol Methods 2012;17(4):510-50.

10. Zeiler FA, Teitelbaum J, West M, et al. The ketamine effect on ICP in traumatic brain injury. Neurocrit Care 2014; 21(1):163-73.

11. Cohen L, Athaide V, Wickham ME, et al. The effect of ketamine on intracranial and cerebral perfusion pressure and health outcomes: a systematic review. Ann Emerg Med 2015; 65(1):43-51.e2. 